\title{
Effects of Stereoregularity on Molecular Parameters of Poly- acrylonitrile Polymerized by Gamma-Ray Irradiation
}

\author{
Kenji KaMIDE, Hitoshi YAMAZAKI, and Yukio MIYAZAKI \\ Fundamental Research Laboratory of Fibers and Fiber-Forming Polymers, \\ Asahi Chemical Industry Company, Ltd., \\ Takatsuki, Osaka 569, Japan
}

(Received March 17, 1986)

\begin{abstract}
Polyacrylonitrile (referred to as $\gamma$-PAN) was prepared by $\gamma$-ray irradiation on an acrylonitrile-urea canal complex at $-78^{\circ} \mathrm{C}$. The pentad tacticity was evaluated from ${ }^{13} \mathrm{C}$ NMR spectra according to a method proposed by Kamide et al. The content of a $\mathrm{mmmm}$ sequence $(\mathrm{m}$; meso) for $\gamma$-PAN was $0.37 \pm 0.02$ over a wide range of molecular weight, which was about 4.4 times larger than that for PAN, prepared by conventional redox polymerization (R-PAN). Light scattering and solution viscosity were measured in dimethyl sulfoxide (DMSO), $57-67 \mathrm{wt} \%$ aq nitric acid at $25^{\circ} \mathrm{C}$. By extrapolation of the second virial coefficient $A_{2}$ to zero $56.5 \mathrm{wt} \%$ aq nitric acid was expected to be a Flory theta solvent at $25^{\circ} \mathrm{C}$. Mark-Houwink-Sakurada equations and empirical relations between the radius of gyration $\left\langle S^{2}\right\rangle_{z}^{1 / 2}$ and $\bar{M}_{w}$ were determined in various solvents. The unperturbed chain dimension $A$ was determined by four methods. In DMSO $\gamma$-PAN has larger $A$ than R-PAN. The conformation parameter $\sigma$ was 2.6-2.8 for $\gamma$-PAN, which is some $35 \%$ larger than the expected value when simple steric hindrance due to the side chain group is considered. Unexpectedly larger conformation parameters $\sigma$ for $\gamma$ - and R-PAN were interpreted by considering the rigidity of the chain due to intra-interactions between neighbouring $\mathrm{CN}$ groups in meso-sequence.
\end{abstract}

KEY WORDS Polyacrylonitrile / Dilute Solution Property / Light

Scattering / Mark-Houwink-Sakurada Equation / Unperturbed Chain

Dimension / Conformation Parameter / $\gamma$-Ray Irradiation / Urea-Canal

Complex / Stereoregularity /

Since the latter 1950's, numerous studies have been carried out on the effects of stereoregularity on the molecular characteristics of polymers in solutions. ${ }^{1-23}$ The results obtained can be summarized in short as: (1) The parameters $K_{m}$ and $a$ in the Mark-HouwinkSakurada (MHS) equation,

$$
[\eta]=K_{m} M^{a}
$$

([ $\eta]$, limiting viscosity number; $M$, molecular weight; $K_{m}$ and $a$, parameters characteristic of the combination of polymer and solvent at a given temperature) are not significantly influenced by the stereoregularity of a polymer, if it is dissolved in a good solvent. 1,7,10,12,17,21,22 (2) The second virial coefficient $A_{2}$ becomes smaller even in a good solvent for a polymer with higher stereoregularity. ${ }^{1,10,17}$ (3) The unperturbed chain dimension $A$ (see eq 5) is larger for a polymer with higher stereoregularity and $A$ of the isotactic polymer is usually larger than $A$ of a syndiotactic polymer. ${ }^{3,6-9,11,14,17,20-23}$ (4) $A$ decreases with an increase in temperature ${ }^{1,2,4-6,20}$ These characteristic features of dilute solutions of stereoregular polymers were reviewed for example by Kamide (1962), ${ }^{24}$ Krigbaum (1964) ${ }^{25}$ and Cowie (1972). ${ }^{26}$ Here, it should be noted that (1) in only less than half the above studies, the tacticities of the polymers were characterized by NMR and other methods as diad (poly(methyl methacrylate) (PMMA), ${ }^{9}$ poly- 
(1-pentene) $(\mathrm{P} 1 \mathrm{P})^{20}$ and triad (poly $(\alpha-$ methyl styrene) (P $\alpha \mathrm{MS}),{ }^{13-16}$ poly(isopropyl acrylate) (PIPA) ${ }^{21,22}$ ); (2) a highly stereospecific polymer has a tendency to form a gel or crystallize when dissolved in theta solvent and $A$ for only a few polymers, such as $\mathrm{P} \alpha \mathrm{MS},{ }^{13,15}$ poly(1-butene) (P1B) ${ }^{18}$ and poly(vinyl acetate) (PVAc), ${ }^{23}$ was determined directly from the unperturbed radius of gyration in the theta solvents by light scattering. (3) For many other polymers, the limiting viscosity number $[\eta]$ or the radius of gyration measured in good solvents was analyzed to estimate $A .^{2,9,14,16,20,22}$ Not much progress has been made for the last ten years.

As is well known, it is experimentally difficult to prepare polyacrylonitrile (PAN) with high stereoregularity and the effect of stereoregularity on the dilute solution properties of PAN has never been studied in detail. A few exceptions are works by Inagaki et $a .^{27}$ and by Kamide and his coworkers. ${ }^{28}$ Inagaki et al. observed that MHS equations and accordingly, A, estimated from Stockmayer-Fixman plot (see eq 8), for PAN in dimethylformamide depend on polymerization temperature when a redox catalyst is used, concluding that the syndio-tacticity, as estimated from the peak at $950 \mathrm{~cm}^{-1}$ in infrared spectrum, might increase with decreasing polymerization temperature $(-30$ $\left.-60{ }^{\circ} \mathrm{C}\right)$. These predictions were not unfortunately confirmed with later NMR experiments by Matsuzaki et al. ${ }^{29,30}$ Kamide, Kobayashi, and their collaborators ${ }^{31}$ studied thermodynamic properties of PAN, polymerized using redox and non-redox catalysts in aqueous and non-aqueous media, noting that the MHS equation and $A$, estimated from the Stockmayer-Fixman plot (see eq 8 ) of the polymers polymerized using azobisisobutyronitrile in benzene, differ slightly, but significantly from those of polymers prepared under different conditions. The detailed structure of the ${ }^{1} \mathrm{H}$ NMR spectrum for these PAN sample are somewhat different, but no further analysis on the tacticity was made in their study. Kamide and Terakawa ${ }^{28}$ who studied the polymer polymerized by irradiating $\gamma$-ray on an acrylonitrile-urea canal complex at low temperature (hereafter, referred to as $\gamma$-PAN), showed that the solubility of $\gamma$-PAN in DMF, a good solvent for PAN conventionally polymerized (hereafter referred as R-PAN), becomes extremely low. Unfortunately, in their work, the diad tacticity for $\gamma$-PAN was indirectly estimated by using Matsuzaki et al.'s method ${ }^{30}$ and the molecular weights for $\gamma$-PAN fractions were indirectly evaluated from the MHS equation, established for R-PAN in a dimethyl sulfoxide (DMSO) solution, assuming that the MHS equation for good solvent is independent of the stereoregularity of the polymer.

Very recently, Kamide and his coworkers $^{32,33}$ succeeded in determining the pentad tacticity of R- and $\gamma$-PANs from ${ }^{13} \mathrm{C}$ NMR spectra in deuterated dimethyl sulfoxide (DMSO- $d_{6}$ ) and disclosed that R-PAN is polymerized according to Bernoulli statistics and $\gamma$-PAN obeys 1st-order Markov statistics.

R-PAN has therefore relatively low stereoregularity as compared with $\gamma$-PAN. The weight fraction of $m m m m$ sequence $(m$; meso configuration) was estimated to be 0.07 for R-PAN and 0.37 for $\gamma$-PAN.

In this article, an attempt was made to investigate the dilute solution properties of $\gamma$-PAN whose stereoregularity is clarified in greater detail and compare the results obtained here with those of R-PAN.

\section{EXPERIMENTAL}

\section{Polymer Samples}

$\gamma$-PAN was polymerized according to the method of White. ${ }^{34}$ Analytical reagent grade urea, manufactured by Kishida Chemicals Co., Osaka, was purified by recrystallization with a mixture of water and methanol $(1: 5$ by weight) and dried in hot air. Inhibitors, added to commercially available acrylonitrile (AN) 
(analytical reagent grade, manufactured by Kishida Chemicals Co.) were extracted completely with a $2 \mathrm{wt} \%$ aq sodium carbonate solution and washed with water. Purified AN was mixed with purified urea to give a $1: 1$ (by weight) mixture, stored in a methanol-dry ice mixture at $-78^{\circ} \mathrm{C}$ for 6 days. The $\mathrm{AN}-$ urea canal complex, formed slowly during storage, was irradiated with $\gamma$-ray from cobalt 60 at $-78^{\circ} \mathrm{C}$ at a dose rate of $3 \times 10^{5} \mathrm{R} \mathrm{h}^{-1}$ for $5 \mathrm{~h}$ (The irradiation dose was $1.5 \mathrm{MR}$.) The polymer was dissolved in DMSO and precipitated with methanol. The polymerization yield was $58 \mathrm{wt}^{\mathrm{o}} \%$. The viscosity-average molecular weight $\bar{M}_{v}$ of the polymer was determined using the MHS equation (in DMSO, Table V) to be $1.70 \times 10^{5}$. For comparison, the R-PAN fractions in the previous paper ${ }^{35}$ were used for light scattering and solution viscosity measurements in DMSO.

\section{NMR Measurements}

${ }^{13} \mathrm{C}$ NMR measurement on $\gamma$-PAN was made on a FT-NMR JEOL FX-400 using deuterated DMSO- $d_{6}$ as solvent at $80^{\circ} \mathrm{C}$. This $\gamma$-PAN polymer was found to have an isotactic triad of $52 \%$, heterotactic triad of $33 \%$ and syndiotactic triad of $15 \%$. The corresponding triads for R-PAN fractions were 26,50 , and $24 \%$, respectively. The pentad tacticity was evaluated by a method previously proposed. ${ }^{32,33}$

\section{Wide-Angle X-Ray Diffraction}

Wide-angle $\mathrm{X}$-ray diffraction for as-polymerized $\gamma$ - and R-PAN whole polymer powders and the precipitated powder of $\gamma$-PAN from the solution was measured on a Rigaku RU 200 PL type X-ray diffraction apparatus.

\section{Solvents}

Reagent-grade DMSO, dimethylacetamide (DMAc), ethylene carbonate (EC), $\gamma$-butyrolactone, and $70 \mathrm{wt} \%$ aq nitric acid, supplied by Kishida Chemicals Co., Osaka, were used as received. The Concentration of nitric acid was adjusted by adding pure water. The R-PAN sample, prepared in the previous paper, ${ }^{35}$ had a residue of the redox catalyst, $\sim \mathrm{SO}_{3} \mathrm{H}$, at the chain end and to suppress the ionization of the end group when dissolved in a solvent, $0.1 \mathrm{wt} \% \mathrm{LiCl}$ was added to the solvents. Since $\gamma$-PAN was synthesized without any catalyst, no $\mathrm{LiCl}$ was necessary.

\section{Solvation}

The adiabatic compressibility of the solution was calculated from the sound velocity and density of the solution and converted to the weight of the solvated solvent per gram of polymer $N_{\mathrm{s}}$ using Passynsky's relation. The sound velocity was measured on a Nusonics Model 6080 Concentration Analyzer at $25 \pm 0.005^{\circ} \mathrm{C}$ in the polymer concentration range of $1-5 \mathrm{~g}$ polymer $/ 100 \mathrm{~g}$ solvent. The details of the measuring conditions have been described already. ${ }^{35}$

\section{Fractionation}

A whole $\gamma$-PAN sample was fractionated by a successive precipitation (SPF) method $^{36}$ using DMSO as a solvent and toluene as a non-solvent. The successive solution method, proved to be theoretically much more effective to obtain sharp fractions, could not be applied here due to extreme difficulty in diluting with DMSO a polymer-rich phase separated after two-phase equilibrium. SPF was conducted under the following conditions: concentration of starting solution, $2 \mathrm{~g} / 100 \mathrm{ml}$; initial concentration, $0.95 \mathrm{~g} / 100 \mathrm{ml}$ for the first fraction; temperature $30^{\circ} \mathrm{C}$; total number of fractions, 20.

\section{Light Scattering}

A Union Giken LS 601 type light scattering photometer was used at $25^{\circ} \mathrm{C}$ for measurements on DMSO solutions. The wave length $\lambda_{0}$ of the polarized He-Ne laser incident light was $633 \mathrm{~nm}$. The experimental data were analyzed by Zimm's procedure to determine the weight-average molecular weight $\bar{M}_{w}$, the 
radius of gyration $\left\langle S^{2}\right\rangle_{z}^{1 / 2}$ and the second virial coefficient $A_{2}$.

A Fica photogoniodiffusiometer 42000 was employed also for aq nitric acid solutions at $\lambda_{0}=546 \mathrm{~nm}$. The details of the experiments and analytical procedure are described elsewhere ${ }^{35}$ and $\left\langle S^{2}\right\rangle_{z}^{1 / 2}$ and $A_{2}$ were evaluated.

The specific refractive index increment $\mathrm{d} \tilde{n}$ / $\mathrm{d} C$ for $\gamma$-PAN was determined to be 0.038 $\mathrm{mlg}^{-1}$ by a Union Giken RM 102 type differential refractiometer at $25^{\circ} \mathrm{C}$ at $\lambda_{0}=633$ $\mathrm{nm}$, which is in an excellent agreement with that for R-PAN $\left(0.038 \mathrm{ml} \mathrm{g}^{-1}\right)$.

\section{Solution Viscosity}

Solution viscosity was measured in DMSO and 57 and $67 \mathrm{wt} \%$ aq nitric acids with a modified Ubbelohde suspension type viscometer at $25 \pm 0.01^{\circ} \mathrm{C}$. [ $\left.\eta\right]$ was determined by Huggins plot.

\section{RESULTS AND DISCUSSION}

Table I shows the pentad tacticity data for three typical $\gamma$-PAN fractions and a whole R-PAN sample. in the table, the data for a whole R-PAN are cited from the previous paper. ${ }^{33}$ The pentad tacticity of $\gamma$-PAN is almost independent of its average-molecular weight. The content of a $\mathrm{mmmm}$ sequence is about 4.8 times larger than that of R-PAN and the content of a rmrm sequence ( $r$, racemo configuration) is only 0.6 times that of R-PAN. These facts indicate that $\gamma$-PAN has a higher stereoregularity than R-PAN.

By applying the pentad method ${ }^{33}$ to the tacticity data, we evaluated the probability of linking a meso sequence to a racemo sequence, $P(m / r)$ and that of linking a racemo sequence to a meso sequence, $P(r / m)$ for each $\gamma$-PAN sample as listed in the bottom lines of Table I. The probability of a meso arrangement $P_{m}$, in Bernoulli statistics, for RPAN was cited from a reference. ${ }^{33}$

With the aid of electric computer, we generated meso- and racemo sequences in PAN
Table I. The observed pentad fractions for $\gamma$-PAN fractions and R-PAN

\begin{tabular}{|c|c|c|c|c|}
\hline \multirow{3}{*}{$\begin{array}{c}\text { Pentad } \\
\text { sequence }\end{array}$} & \multicolumn{3}{|c|}{$\gamma$-PAN } & R-PAN \\
\hline & \multicolumn{3}{|c|}{$\bar{M}_{w} \times 10^{-4}$} & \\
\hline & 9.8 & 26.3 & 34.8 & $18.8^{\mathrm{a}}$ \\
\hline $\mathrm{mmmm}$ & 0.387 & 0.386 & 0.350 & 0.077 \\
\hline$m m m r$ & 0.125 & 0.112 & 0.108 & 0.125 \\
\hline$r m m r$ & 0.042 & 0.034 & 0.043 & 0.068 \\
\hline mmrm & 0.117 & 0.120 & 0.118 & 0.125 \\
\hline$m m r r$ & 0.068 & 0.074 & 0.076 & 0.097 \\
\hline $\mathrm{rmrm}$ & 0.083 & 0.082 & 0.096 & 0.149 \\
\hline$r m r r$ & 0.050 & 0.052 & 0.063 & 0.129 \\
\hline mrrm & 0.029 & 0.028 & 0.030 & 0.055 \\
\hline$m r r r$ & 0.045 & 0.055 & 0.052 & 0.112 \\
\hline \multirow[t]{2}{*}{$r r r r$} & 0.054 & 0.056 & 0.062 & 0.065 \\
\hline & \multicolumn{4}{|c|}{ Average } \\
\hline$P(r / m)$ & 0.191 & 0.189 & 0.210 & \\
\hline$P(m / r)$ & 0.536 & 0.509 & 0.508 & \\
\hline
\end{tabular}

a Reference 33 .

molecules with the degree of polymerization $(N=4001)$ by Monte Carlo method under the following conditions: $P(m / r)=0.518$, $P(r / m)=0.197$ for $\gamma$-PAN and $P_{m}=0.519$ for R-PAN.

We define the number of "blocks" for $i$ th molecule, which consists of $p$ of meso sequences, $m_{1} \cdots m_{p}$, as $F_{m}^{i}(p)$ and the number of "blocks" for the same molecule, which consists of $q$ of racemo sequences, $r_{1} \cdots r_{q}$, as $F_{r}^{i}(q)$, respectively (see Figure 1$)$. Then, $F_{m}^{i}(p)$ and $F_{r}^{i}(q)$ for $i$-th molecule can be averaged over all chains $(i=1 \sim \mathrm{I})$ comprising the sample and denoted by $\overline{F_{m}(p)}$ and $\overline{F_{r}(q)}$, respectively. $\overline{F_{m}(p)}$ and $\overline{F_{r}(q)}$ are given by

$$
\left.\begin{array}{l}
\overline{F_{m}(p)}=\frac{\sum_{i=1}^{I} F_{m}^{i}(p)}{I} \\
\overline{F_{r}(q)}=\frac{\sum_{i=1}^{I} F_{r}^{i}(q)}{I}
\end{array}\right\}
$$

When $I$ is sufficiently large, equation 2 reduces to equation $2^{\prime}$ 
Effects of Stereoregularity on Molecular Parameters of PAN

Meso- racemo-sequence

iNumber of meso and racemo "block"

$F_{m}^{\mathfrak{l}}(p)$ and $F_{r}^{i}(q)$
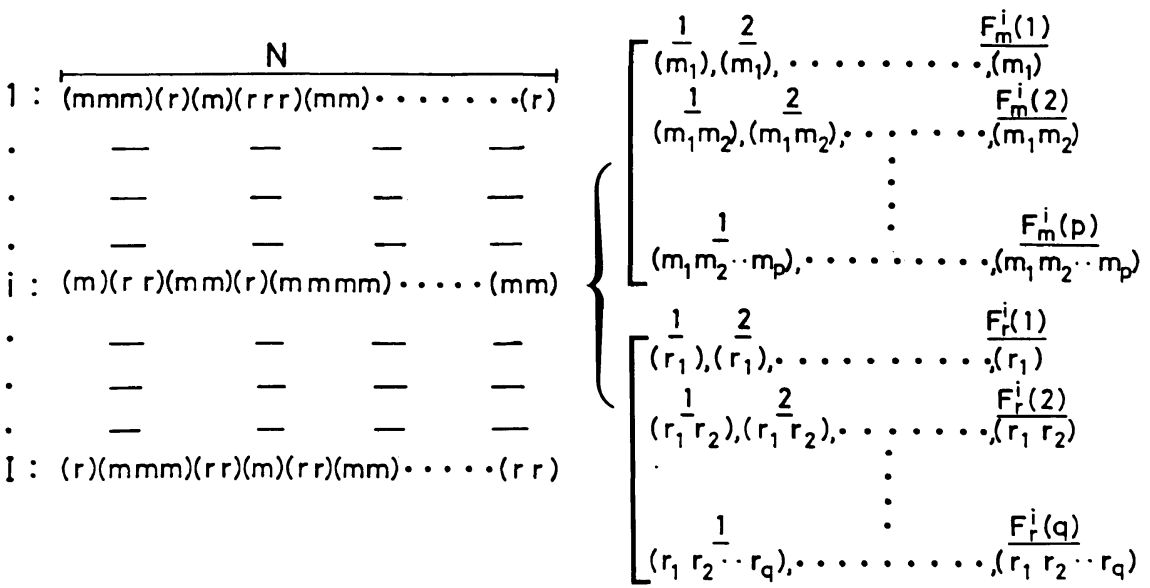

Figure 1. Meso- and racemo-sequence distribution of polyacrylonitrile and the number of "blocks" $F_{m}^{i}(p)$ or $F_{r}^{i}(n)$

Table II. Distribution of meso- and racemo-sequence of $\gamma$-PAN and R-PAN $\left(M=21.2 \times 10^{4}\right)$

\begin{tabular}{llllllllllllllllllllllllllllllllllllllllll}
\hline Polymer & $p$ or $q$ & 1 & 2 & 3 & 4 & 5 & 6 & 7 & 8 & 9 & 10 & 11 & 12 & 13 & 14 & 15 & 16 & 17 & 18 & 19 & 20 & 21 & 22 & 23 & 24 & 25 \\
\hline \multirow{2}{*}{-PAN } & $\overline{F_{m}(p)}$ & 114 & 92 & 73 & 59 & 47 & 38 & 30 & 24 & 20 & 16 & 13 & 10 & 8 & 7 & 5 & 4 & 3 & 3 & 2 & 2 & 1 & 1 & 1 & 1 & 1 \\
& $F_{r}(q)$ & 301 & 143 & 68 & 33 & 16 & 7 & 4 & 2 & 1 & 1 & 0 & 0 & 0 & 0 & 0 & 0 & 0 & 0 & 0 & 0 & 0 & 0 & 0 & 0 & 0 \\
\hline \multirow{2}{*}{ R-PAN } & $\overline{F_{m}(p)}$ & 480 & 249 & 129 & 67 & 35 & 18 & 9 & 5 & 3 & 2 & 1 & 1 & 0 & 0 & 0 & 0 & 0 & 0 & 0 & 0 & 0 & 0 & 0 & 0 & 0 \\
& $F_{r}(q)$ & 517 & 249 & 120 & 58 & 28 & 13 & 6 & 3 & 2 & 1 & 1 & 0 & 0 & 0 & 0 & 0 & 0 & 0 & 0 & 0 & 0 & 0 & 0 & 0 & 0
\end{tabular}

$$
\left.\begin{array}{l}
\overline{F_{m}(p)}=B_{m} P_{\mathrm{A}} P_{\mathrm{B}}^{p-1} \\
\overline{F_{r}(q)}=B_{r} P_{\mathrm{C}} P_{\mathrm{D}}^{q-1}
\end{array}\right\}
$$

Here, $B_{m}$ (or $B_{r}$ ) is the total number of meso(or racemo-) sequence blocks in the molecule, and expressed as,

$$
\left.\begin{array}{l}
B_{m}=\sum_{i=1}^{I} \sum_{p=1}^{N-1} F_{m}^{i}(p) / I \\
B_{r}=\sum_{i=1}^{I} \sum_{q=1}^{N-1} F_{r}^{i}(q) / I
\end{array}\right\}
$$

$P_{\mathrm{A}}=P(r / m), P_{\mathrm{B}}=1-P(r / m), P_{\mathrm{C}}=P(m / r)$, and $P_{\mathrm{D}}=1-P(m / r)$ for $\gamma$-PAN and $P_{\mathrm{A}}=1-P_{m}$, $P_{\mathrm{B}}=P_{m}, \quad P_{\mathrm{C}}=P_{m}, \quad$ and $P_{\mathrm{D}}=1-P_{m}$ for RPAN.
$\overline{F_{m}(p)}$ and $\overline{F_{r}(q)}$ for all possible $p$ or $q$ for $\gamma$ - and R-PAN were calculated using eq $2^{\prime}$ and 3 from $P(r / m)$ and $P(m / r)$ and $P_{m}$ values in Table $I$ and putting $I=100$ and $N=4001$ (i.e., molecular weight $M=2.12 \times$ $\left.10^{5}\right)$. The results are summarized in Table II. The details of calculation will be published elsewhere. ${ }^{37}$ Of course, $F_{n}^{i}(p)$ and $F_{r}^{i}(q)$ have some distributions around $\overline{F_{m}(p)}$ and $\overline{F_{r}(q)}$, respectively.

We chose six solvents among good solvents, widely used hitherto, for R-PAN to examine solvent power against $\gamma$-PAN. For this purpose, four grams of the whole polymer sample $\left(\bar{M}_{v}=1.70 \times 10^{5}\right)$ were mixed with $100 \mathrm{ml}$ of solvent at $60^{\circ} \mathrm{C}$ for $5 \mathrm{~h}$. Aspolymerized $\gamma$-PAN dissolved completely 
after mixing in both DMSO and $67 \mathrm{wt} \%$ aq nitric acid, but only $0.2 \mathrm{wt} \%$ solutions were obtained in DMF, DMAc, EC, and $\gamma$-butyrolactone. In the latter group of solvents, the undissolved polymer did not even swell. When powder-like $\gamma$-PAN, precipitated with methanol from $4 \mathrm{~g} / 100 \mathrm{ml}$ solution of aspolymerized polymer in DMSO, was mixed again at $60^{\circ} \mathrm{C}$ with DMAc, the solubility of the polymer increased remarkably up to $3.62 \mathrm{wt}^{\mathrm{o}} \%$. The stereoregularity of the dissolved and undissolved parts of $\gamma$-PAN precipitate/DMAc mixture, isolated by centrifuging under $4.9 \times 10^{4} \mathrm{G}$ for $1 \mathrm{~h}$, was estimated by ${ }^{13} \mathrm{C}$ NMR method in DMSO- $d_{6}$. It was confirmed that there was only slight a difference in the stereoregularity between two parts: isotactic triad, $48.6 \%$, heterotactic triad, $37.3 \%$, and syndiotactic triad, $14.1 \%$ for the soluble part, and 53.3, 33.6, and 13.1 $\%$ for the insoluble part.

Figure 2 shows X-ray diffraction patterns of as-polymerized and precipitated powders from DMSO solution of unfractionated $\gamma$ PAN and R-PAN samples. As-polymerized $\gamma$ PAN has a higher crystallinity and larger crystalline size than precipitated $\gamma$-PAN and as-polymerized R-PAN. This strongly suggests that the crystallinity, besides the stereoregularity, governs mainly the solubility of PAN in solvents.

Figure 3 shows $A_{2}$, as determined by the light scattering method, of solutions of a $\gamma$ PAN fraction (Sample cord $\gamma-1-3$ ) dissolved in 57, 59, 62 and $67 \mathrm{wt} \%$ aq nitric acid at $25^{\circ} \mathrm{C}$. The sample had $\bar{M}_{w}$ of $1.68 \times 10^{5}$, determined by the light scattering method in DMSO. The nitric acid concentration of a solvent, in which $A_{2}$ becomes zero at $25^{\circ} \mathrm{C}$, can be extrapolated from Figure 3 to be 56.5 $\mathrm{wt} \%$. That is, $56.5 \mathrm{wt} \%$ aq nitric acid can be regarded as a Flory's theta solvent of $\gamma$-PAN at $25^{\circ} \mathrm{C}$. Kamide et al. determined the Flory's theta solvent of R-PAN to be $55 \mathrm{wt}^{\mathrm{o}} \%$ aq nitric acid at $25^{\circ} \mathrm{C} .{ }^{35}$ Then, theta solvent for $\gamma$-PAN is slightly higher in nitric acid con-

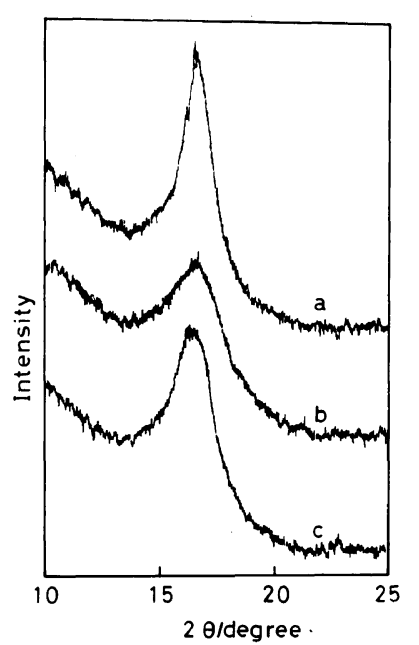

Figure 2. X-Ray diffraction patterns: a) as-polymerized $\gamma$-PAN; b) precipitated powder of $\gamma$-PAN from DMSO solution; c) as-polymerized R-PAN.

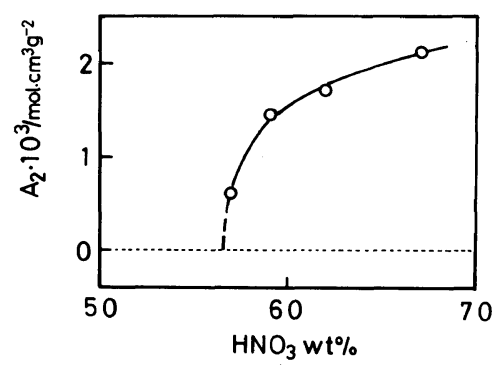

Figure 3. Variation in the second virial coefficient $A_{2}$ of a $\gamma$-PAN fraction $(\gamma-1-3)$ with nitric acid concentration.

centration than that for R-PAN. The light scattering measurement on $\gamma$-PAN in 56.5 $\mathrm{wt} \%$ aq nitric acid was attempted, but was unsuccessful due to experimental difficulty in exclusion of gel-like materials during filtration.

Table III shows $\bar{M}_{w},\left\langle S^{2}\right\rangle_{z}^{1 / 2}, A_{2}$ and [ $\left.\eta\right]$ of eight $\gamma$-PAN fractions in DMSO, 67 and 57 $\mathrm{wt} \%$ aq nitric acids. Table IV summarizes similar data for R-PAN in DMSO at $25^{\circ} \mathrm{C}$. In the table, $\bar{M}_{w}$ data in DMF in the previous paper $^{35}$ are tabulated for comparison. Table IV indicates that $\bar{M}_{w}$ values obtained for RPAN in DMSO are in good agreement with those in DMF within an experimental un- 
Table III. Results of light scattering and limiting viscosity number for $\gamma$-PAN fractions in various solvents at $25^{\circ} \mathrm{C}$

\begin{tabular}{|c|c|c|c|c|c|c|c|c|}
\hline \multirow{2}{*}{ Sample } & \multirow{2}{*}{$\frac{\bar{M}_{w} \times 10^{-4}}{\text { DMSO }}$} & \multicolumn{3}{|c|}{$\left\langle S^{2}\right\rangle_{z}^{1 / 2} \times 10^{8} / \mathrm{cm}$} & \multirow{2}{*}{$\frac{A_{2} \times 10^{3 \mathrm{a}}}{\mathrm{DMSO}}$} & \multicolumn{3}{|c|}{$[\eta] / \mathrm{cm}^{3} \mathrm{~g}^{-1}$} \\
\hline & & DMSO & $67 \mathrm{wt} \% \mathrm{HN}$ & $\mathrm{wt} \% \mathrm{HNO}_{3}$ & & DMSO & $67 \mathrm{wt} \% \mathrm{HNC}$ & $\mathrm{wt} \% \mathrm{HNO}_{3}$ \\
\hline$\gamma-1-1$ & 7.1 & 152 & 145 & 143 & 0.79 & 135 & 130 & 100 \\
\hline$\gamma-1-2$ & 10.7 & 193 & 189 & 176 & 0.83 & 166 & 168 & 125 \\
\hline$\gamma-1-3$ & 16.8 & 242 & 243 & 220 & 0.59 & 224 & 216 & 161 \\
\hline$\gamma-1-4$ & 26.3 & 317 & 312 & 275 & 0.60 & 275 & 272 & 182 \\
\hline$\gamma-1-5$ & 34.8 & 348 & 359 & 317 & 0.53 & 347 & 329 & 222 \\
\hline$\gamma-1-6$ & 51.2 & 443 & - & - & 0.40 & 408 & 396 & - \\
\hline$\gamma-1-7$ & 61.5 & 507 & 489 & 432 & 0.50 & 473 & 460 & 300 \\
\hline$\gamma-1-8$ & 78.3 & 544 & 565 & 487 & 0.47 & 540 & 520 & 342 \\
\hline
\end{tabular}

${ }^{\mathrm{a}} \mathrm{cm}^{3} \mathrm{molg}^{-2}$.

Table IV. Results of light scattering and limiting viscosity number for R-PAN fractions in DMF and DMSO at $25^{\circ} \mathrm{C}$

\begin{tabular}{|c|c|c|c|c|c|}
\hline \multirow{2}{*}{ Sample } & \multicolumn{2}{|c|}{$\bar{M}_{w} \times 10^{-4}$} & \multirow{2}{*}{$\begin{array}{c}\left\langle S^{2}\right\rangle_{z}^{1 / 2} \times \\
10^{8 \mathrm{~b}}\end{array}$} & \multirow{2}{*}{$\begin{array}{c}A_{2} \times \\
10^{3 \mathrm{c}} \\
\text { DMSO }\end{array}$} & \multirow{2}{*}{$\frac{[\eta]^{\mathrm{d}}}{\mathrm{DMSO}}$} \\
\hline & $\mathrm{DMF}^{\mathrm{a}}$ & DMSO & & & \\
\hline PAN-1 & 5.2 & - & - & - & 100 \\
\hline PAN-2 & 7.5 & - & - & - & 124 \\
\hline PAN-3 & 10.7 & 10.0 & 185 & 1.60 & 146 \\
\hline PAN-4 & 15.8 & - & - & - & 190 \\
\hline PAN-5 & 21.5 & 22.0 & 269 & 1.15 & 226 \\
\hline PAN-6 & 31.2 & 33.3 & 329 & 0.70 & 278 \\
\hline PAN-7 & 52.0 & 50.8 & 438 & 0.82 & 400 \\
\hline
\end{tabular}

${ }^{a}$ Reference $35 .{ }^{b} \mathrm{~cm} .{ }^{\mathrm{c}} \mathrm{cm}^{3} \mathrm{~mol} \mathrm{~g}^{-2} .{ }^{\mathrm{d}} \mathrm{cm}^{3} \mathrm{~g}^{-1}$.

certainty $( \pm 7 \%)$ and R-PAN molecule dissolves molecularly in DMSO and DMF.

Figure 4 shows the $\log -\log$ plot of $[\eta]$ against $\bar{M}_{w}$ of $\gamma$-PAN and R-PAN in DMSO and aq nitric acids. Here, the data of R-PAN in aq nitric acid are cited from the previous paper. ${ }^{35}$ MHS equations were evaluated using the least-squares method and the results are summarized, together with those for R-PAN in various solvents in literature, ${ }^{35}$ in Table V. In DMSO solutions, $[\eta]$ for $\gamma-\mathrm{PAN}$ is significantly larger than that for R-PAN if the samples with the same $\bar{M}_{w}$ are compared. In contrast to this, in $67 \mathrm{wt} \%$ aq nitric acid $[\eta]$ of R-PAN is just slightly larger than that of

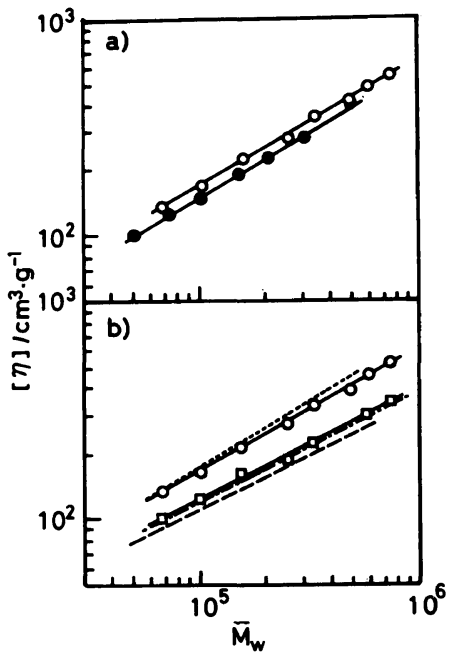

Figure 4. Log-log plots of the limiting viscosity number $[\eta]$ versus the weight-average molecular weight $\bar{M}_{w}$ for $\gamma$-PAN and R-PAN. a) $\bigcirc, \gamma$-PAN in DMSO;, $\mathrm{R}$ PAN in DMSO. b) $\bigcirc, \gamma$-PAN in $67 \mathrm{wt} \%$ aq $\mathrm{HNO}_{3} ; \square$, $\gamma$-PAN in $57 \mathrm{wt} \%$ aq $\mathrm{HNO}_{3}$; dot-dash line, $\gamma$-PAN in $56.5 \mathrm{wt} \%$ aq $\mathrm{HNO}_{3}$; dotted line, R-PAN in $67 \mathrm{wt} \%$ aq $\mathrm{HNO}_{3}$; broken line R-PAN in $55 \mathrm{wt} \%$ aq $\mathrm{HNO}_{3}$.

$\gamma$-PAN if $\bar{M}_{w}>2 \times 10^{4}$, but the difference is insignificant. The effect of stereospecificity becomes significant with the MHS equation in DMSO, but not in aq nitric acid.

[ $\eta$ ] of a $\gamma$-PAN solution in $57 \mathrm{wt} \%$ aq nitric acid $(a=0.503)$, is in the vicinity of the theta solvent and about $15 \%$ larger than that of $\mathrm{R}$ PAN in $55 \mathrm{wt} \%$ aq nitric acid, in which 
$A_{2}=0$, if the same $\bar{M}_{w}$ are compared.

Using the experimental relation between [ $\eta]$ and the concentration of nitric acid, the $[\eta]$ value in a $56.5 \mathrm{wt} \%$ nitric acid was extrapolated and the following MHS relation was obtained:

$$
[\eta]=0.365 \bar{M}_{w}^{0.50_{1}}\left(\mathrm{~cm}^{3} \mathrm{~g}^{-1}\right)
$$

The exponent in eq a coincides well with 0.5 , as anticipated if $56.5 \mathrm{wt} \%$ aq nitric acid is a theta solvent. Equation a is shown as a dotdash line in Figure 4b. In theta solvents, $[\eta]$ of $\gamma$-PAN is some $8.5 \%$ larger than that of RPAN, if the samples have the same $\bar{M}_{w}$. In a good solvent, such as $67 \mathrm{wt} \%$ aq nitric acid, MHS equations are not significantly influenced by the stereoregularity of PAN, but in a poor solvent, the situation is different.

Kamide and Terakawa ${ }^{28}$ observed that $\gamma$ PAN dissolves in ethylene carbonate at $60^{\circ} \mathrm{C}$, in which the exponent $a$ in MHS equation equals with 0.5 :

$$
[\eta]=0.295 \bar{M}_{v}^{0.50}\left(\mathrm{~cm}^{3} \mathrm{~g}^{-1}\right)
$$

Here, $\bar{M}_{v}$ in eq a' was evaluated using MHS eq obtained by Kamide and Terakawa ${ }^{28}$ for RPAN in DMSO, assuming that the above equation is valid independent of stereoregularity. The polymer prepared in this study dissolved only partially in EC and the polymer-solvent mixture became obviously turbid and any solution viscosity measurements were impossible. Then, some significant differences in the stereospecificity and/or the crystallinity should be considered between two $\gamma$-PAN polymers synthesized in KamideTerakawa's $^{28}$ and the present work.

Figure 5 shows the $\log -\log$ plot of $\left\langle S^{2}\right\rangle_{z}^{1 / 2}$ and $\bar{M}_{w}$ of the $\gamma$-PAN and R-PAN in DMSO and in aq nitric acids. The semi-empirical relation between $\left\langle S^{2}\right\rangle_{z}^{1 / 2}$ and $\bar{M}_{w}$ is in the form,

$$
\left\langle S^{2}\right\rangle_{z}^{1 / 2}=K_{\gamma} \bar{M}_{w}^{\gamma}
$$

and was determined by the least-squares method and the parameters $K_{\gamma}$ and $\gamma$ evaluated

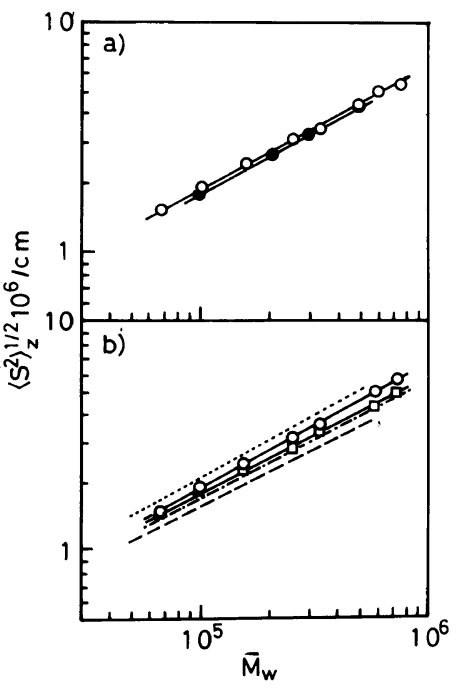

Figure 5. Plots of the radius of gyration $\left\langle S^{2}\right\rangle_{z}^{1 / 2}$ as a function of the weight-average molecular weight $\bar{M}_{w}$ for $\gamma$-PAN and R-PAN. Marks have the same meaning as those in Figure 4.

Table V. $K_{m}$ and $a$ in Mark-Houwink-Sakurada equations and $K_{\gamma}$ and $\gamma$ in relations between

\begin{tabular}{|c|c|c|c|c|c|}
\hline Polymer & Solvent & $K_{m}$ & $a$ & $\begin{array}{c}K_{\gamma} \times \\
10^{8}\end{array}$ & $\gamma$ \\
\hline \multirow[t]{4}{*}{$\gamma$-PAN } & DMSO & 0.204 & $0.58_{0}$ & 0.378 & 0.54 \\
\hline & $67 \mathrm{wt} \% \mathrm{HNO}_{3}$ & 0.217 & $0.57_{3}$ & 0.320 & 0.55 \\
\hline & $57 \mathrm{wt} \% \mathrm{HNO}_{3}$ & 0.363 & $0.50_{3}$ & 0.472 & 0.51 \\
\hline & $56.5 \mathrm{wt} \% \mathrm{HNO}_{3}$ & 0.365 & $0.50_{1}$ & 0.474 & 0.51 \\
\hline \multirow[t]{5}{*}{ R-PAN } & DMSO & 0.153 & $0.60_{0}$ & 0.337 & 0.54 \\
\hline & $67 \mathrm{wt} \% \mathrm{HNO}_{3}{ }^{\mathrm{a}}$ & 0.122 & $0.62_{2}$ & 0.359 & 0.55 \\
\hline & $55 \mathrm{wt} \% \mathrm{HNO}_{3}{ }^{\mathrm{a}}$ & 0.342 & $0.50_{1}$ & 0.480 & 0.50 \\
\hline & $\mathrm{DMF}^{\mathrm{a}}$ & 0.052 & $0.69_{0}$ & 0.317 & 0.55 \\
\hline & $85 w t^{\circ} \% \mathrm{EC}^{\mathrm{a}}$ & 0.256 & 0.49 & 0.431 & 0.50 \\
\hline
\end{tabular}
$\left\langle S^{2}\right\rangle_{z}^{1 / 2}$ and $\bar{M}_{w}$ for $\gamma$-PAN and R-PAN in various solvents at $25^{\circ} \mathrm{C}$

a Reference 35 .

thus are compiled in the fifth and sixth columns of Table V. $\left\langle S^{2}\right\rangle_{z}^{1 / 2}$ of $\gamma$-PAN is larger than that of R-PAN, when both polymers with constant $\bar{M}_{w}$ are dissolved in DMSO, but the former is very slightly smaller in a $67 \mathrm{wt} \%$ aq nitric acid than the latter. Same tendency was also observed in MHS relations of these polymer solutions. Using the dependence of 
$\left\langle S^{2}\right\rangle_{z}^{1 / 2}$ on the concentration of nitric acid, as illustrated in Figure 6, we estimated $\left\langle S^{2}\right\rangle_{z}^{1 / 2}$ value of $\gamma$-PAN in $56.5 \mathrm{wt} \%$ aq nitric acid (that is, $\left\langle S^{2}\right\rangle_{0, z}^{1 / 2}$, the suffix o denotes the unperturbed state) and obtained

$$
\left\langle S^{2}\right\rangle_{\mathrm{o}, z}^{1 / 2}=0.474 \times 10^{-8} \bar{M}_{w}^{0.51}(\mathrm{~cm})
$$

The relation (b) is shown in Figure $5 \mathrm{~b}$ as a dot-dash line. In theta solvents, $\left\langle S^{2}\right\rangle_{z}^{1 / 2}$ of $\gamma$-PAN is larger than that of R-PAN if the same $\bar{M}_{w}$ are compared. In other words, the effect of the stereoregularity on $\left\langle S^{2}\right\rangle_{z}^{1 / 2} v s . \bar{M}_{w}$ relations is significant in a theta solvent.

Figure 7 shows the Flory's viscosity parameter $\Phi\left(\equiv[\eta] \bar{M}_{w} q_{w, z} / 6^{3 / 2}\left\langle S^{2}\right\rangle_{z}^{3 / 2}, q_{w, z}\right.$, correction factor for polydispersity, was calculated assuming the Schulz-Zimm distribution and the ratio of weight- to number-average molecular weight $\bar{M}_{w} / \bar{M}_{n}(=1.3)^{35}$ for $\gamma$-PAN and R-PAN plotted as a function of $\bar{M}_{w}$ for the solutions of $\gamma$-PAN in DMSO, $67 \mathrm{wt} \%$ aq nitric acid and $57 \mathrm{wt} \%$ aq nitric acid and the R-PAN in DMSO. $\Phi$ values remain nearly constant, giving an averaged value of (2.6 \pm $0.3) \times 10^{23}$. There is no significant difference in $\Phi$ between $\gamma$ - and R-PAN.

Figure 8 shows the plot of $A_{2}$ as a function of $\bar{M}_{w}$ for $\gamma$-PAN and R-PAN in DMSO at $25^{\circ} \mathrm{C}$. As expected $A_{2}$ for $\gamma$-PAN is smaller than that for R-PAN.

The unperturbed chain dimension $A$ ( $\equiv$ $\left(\left\langle R^{2}\right\rangle_{\mathrm{o}} / M\right)^{1 / 2},\left\langle R^{2}\right\rangle_{\mathrm{o}}^{1 / 2}$, the mean-square endto-end distance of the unperturbed chain) was determined by using the following methods:

\section{Method $2 A$}

$\left\langle S^{2}\right\rangle_{z}^{1 / 2}$ of a polymer in a Flory theta solvent (in this case, $56.5 \mathrm{wt} \%$ aq nitric acid at $25^{\circ} \mathrm{C}$ ) allows $\mathrm{A}$ to be determined from

$$
A=6^{1 / 2}\left(\left\langle S^{2}\right\rangle_{0} / M\right)^{1 / 2}
$$

\section{Method 2B}

$$
A=6^{1 / 2}\left(\left\langle S^{2}\right\rangle_{\mathrm{o}} / M\right)^{1 / 2}=6^{1 / 2}\left(\left\langle S^{2}\right\rangle / M \alpha_{\mathrm{s}}^{2}\right)^{1 / 2}
$$

$\alpha_{\mathrm{s}}$ is the linear expansion factor, calculated

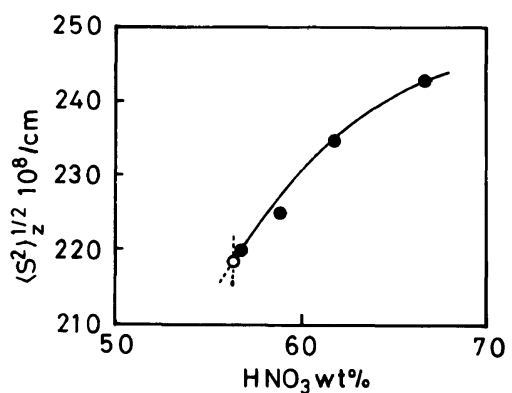

Figure 6. Variation in the radius of gyration $\left\langle S^{2}\right\rangle_{z}^{1 / 2}$ with nitric acid concentration for a $\gamma$-PAN fraction $(\gamma-1$ 3). - experimental data; $\bigcirc$, extrapolated point to a theta solvent.

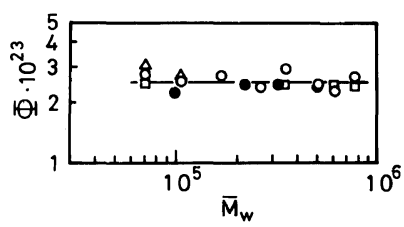

Figure 7. Molecular weight dependence of Flory's viscosity parameter $\Phi$. $\bigcirc, \gamma$-PAN in DMSO; $\square, \gamma$-PAN in $57 \mathrm{wt} \%$ aq. $\mathrm{HNO}_{3} ; \triangle, \gamma-\mathrm{PAN}$ in $67 \mathrm{wt} \%$ aq $\mathrm{HNO}_{3} ;$ R-PAN in DMSO.

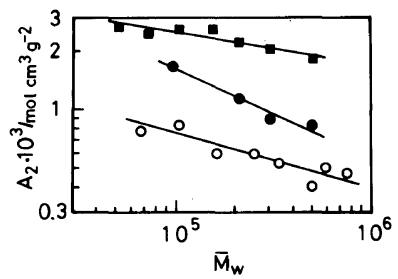

Figure 8. Molecular weight dependence of the second virial coefficient $A_{2}$ for $\gamma$-PAN in DMSO (O), R-PAN in DMSO (O), and R-PAN in DMF

using the penetration function $\psi$ from $A_{2}, \bar{M}_{w}$, and $\left\langle S^{2}\right\rangle_{z}^{1 / 2}$ data. Here, Kurata-FukatsuSotobayashi-Yamakawa theory, ${ }^{38}$ connecting $\psi$ with the excluded volume parameter $z$ and the Fixman theory ${ }^{39}$ of the excluded volume effect are utilized.

\section{Method 2C (Baumann plot $)^{40}$}

$\left(\left\langle S^{2}\right\rangle / M\right)^{3 / 2}=A^{3} / 6^{3 / 2}+1 /\left(4 \pi^{3 / 2}\right) B M^{1 / 2}$

where $B$ is the long-range interaction parameter. 


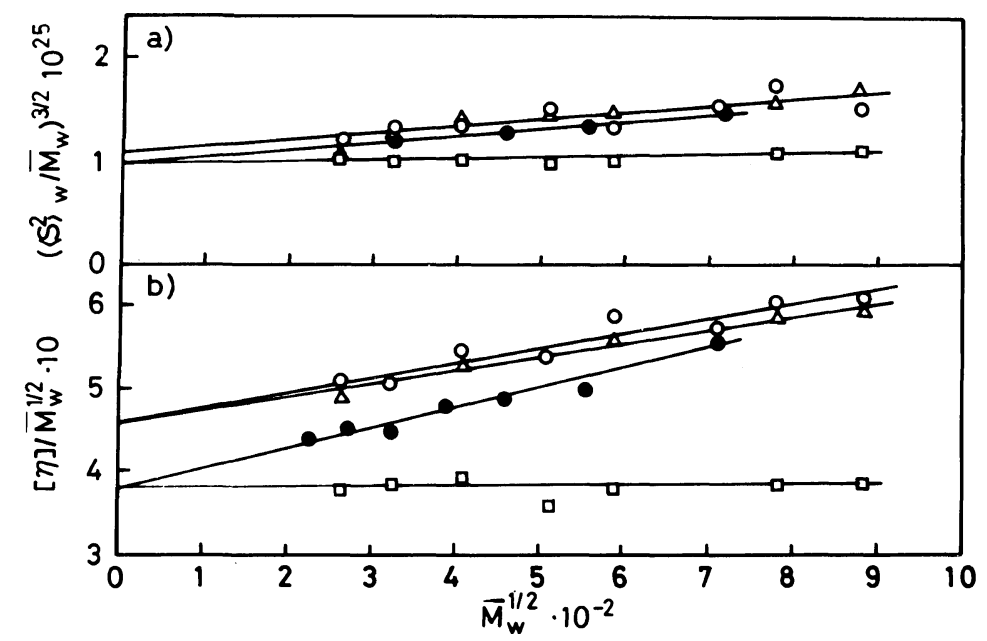

Figure 9. a) Baumann plot (method 2C), b) Stockmayer-Fixman Plot (method 2E): $\bigcirc, \gamma$-PAN in DMSO; $\triangle, \gamma$-PAN in $67 \mathrm{wt} \%$ aq $\mathrm{HNO}_{3} ; \square, \gamma-\mathrm{PAN}$ in $57 \mathrm{wt} \%$ aq $\mathrm{HNO}_{3} ; \boldsymbol{O}, \mathrm{R}-\mathrm{PAN}$ in DMSO.

Table VI. Unperturbed chain dimension $A$, long-range interaction parameter $B$, conformation parameter $\sigma$ and characteristic ratio $C_{\infty}$ of $\gamma$-PAN and $\mathrm{R}-\mathrm{PAN}$ in various solvents at $25^{\circ} \mathrm{C}$

\begin{tabular}{|c|c|c|c|c|c|c|c|c|c|c|c|}
\hline \multirow{2}{*}{ Polymer } & \multirow{2}{*}{ Solvent } & \multicolumn{6}{|c|}{$A \times 10^{8} / \mathrm{cm}$} & \multirow{2}{*}{$\sigma$} & \multirow{2}{*}{$C_{\infty}$} & \multicolumn{2}{|c|}{$B \times 10^{27} / \mathrm{cm}^{3}$} \\
\hline & & $2 \mathrm{~A}$ & $2 \mathrm{~B}$ & $2 \mathrm{C}$ & $2 \mathrm{E}$ & $2 \mathrm{~F}$ & $\begin{array}{c}\text { Most } \\
\text { probable }\end{array}$ & & & $2 \mathrm{C}$ & $2 \mathrm{E}$ \\
\hline \multirow[t]{4}{*}{$\gamma$-PAN } & DMSO & - & 1.19 & 1.17 & 1.17 & 1.20 & 1.18 & 2.80 & 15.5 & 1.18 & 0.94 \\
\hline & $67 \mathrm{wt} \% \mathrm{HNO}_{3}$ & - & - & 1.17 & 1.17 & 1.20 & 1.18 & 2.80 & 15.5 & 1.18 & 0.91 \\
\hline & $57 \mathrm{wt} \% \mathrm{HNO}_{3}$ & - & - & 1.12 & 1.10 & 1.09 & 1.10 & 2.61 & 13.5 & 0.09 & 0.01 \\
\hline & $56.5 \mathrm{wt} \% \mathrm{HNO}_{3}$ & 1.13 & - & 1.12 & 1.08 & 1.08 & 1.10 & 2.61 & 13.5 & 0 & 0 \\
\hline \multirow[t]{5}{*}{ R-PAN } & DMSO & - & - & 1.12 & 1.09 & 1.10 & 1.10 & 2.61 & 13.5 & 1.51 & 1.19 \\
\hline & $67 \mathrm{wt}_{0}^{\circ} / \mathrm{HNO}_{3}{ }^{\mathrm{a}}$ & - & - & 1.29 & 1.29 & 1.30 & 1.29 & 3.06 & 18.5 & 3.17 & 2.69 \\
\hline & $55 \mathrm{wt} \% \mathrm{HNO}_{3}{ }^{\mathrm{a}}$ & 1.06 & - & 1.06 & 1.06 & 1.06 & 1.06 & 2.51 & 12.6 & 0 & 0 \\
\hline & $\mathrm{DMF}^{\mathrm{a}}$ & - & - & 1.09 & 1.05 & 1.05 & 1.06 & 2.51 & 12.6 & 2.33 & 2.32 \\
\hline & $85 w t^{\circ} / \mathrm{EC}^{\mathrm{a}}$ & 0.95 & - & 0.95 & 0.96 & 0.96 & 0.95 & 2.25 & 10.1 & 0 & 0 \\
\hline
\end{tabular}

a Reference 35.

Method 2E (Stockmayer-Fixman plot $)^{41}$

$$
[\eta] / M^{1 / 2}=K+2(3 / 2 \pi)^{3 / 2} \Phi_{0}(\infty) B M^{1 / 2}
$$

with

$$
K=\Phi_{0}(\infty) A^{3}
$$

and

$$
\Phi_{0}(\infty)=2.87 \times 10^{23}
$$

Method $2 F$ (Kamide-Moore plot) ${ }^{42}$

$$
\begin{gathered}
-\log K_{m}+\log \left[1+2\left\{(a-0.5)^{-1}-2\right\}^{-1}\right] \\
=-\log K+(a-0.5) \log M_{0}
\end{gathered}
$$

(9) where $M_{0}$ can be approximated with a geometrical average of the upper and lower limits of molecular weights, in which MHS eq is applicable.

Figure 9a and $\mathrm{b}$ show Baumann (Method 
2C) and Stockmayer-Fixman (Method 2E) plots of $\gamma$-PAN (open mark) and R-PAN (closed mark) fractions. The intercept and slope of these plots enable us to estimate $A$ and $B$, respectively.

Table VI summarizes $A$ and $B$ values estimated thus and $A$ values evaluated by methods $2 \mathrm{~B}$ and $2 \mathrm{~F}$. Then, we can regard as an average of the $A$ values estimated by methods $2 \mathrm{~A}, 2 \mathrm{~B}, 2 \mathrm{C}, 2 \mathrm{E}$, and $2 \mathrm{~F}$ as the most probable value shown in the 8th column of the table. The table contains also the $A$ values of R-PAN in 67 and $55 \mathrm{wt} \%$ aq nitric acid, DMF and $85 \mathrm{wt} \%$ EC for comparison. ${ }^{35}$

In DMSO, $A(\gamma-\mathrm{PAN})>A(\mathrm{R}-\mathrm{PAN})$ and $B(\gamma$-PAN $)<B(\mathrm{R}-\mathrm{PAN})$ hold and in consequence, $[\eta](\gamma-\mathrm{PAN})>[\eta](\mathrm{R}-\mathrm{PAN})$. In $67 \mathrm{wt} \%$ aq nitric acid, $A(\gamma-\mathrm{PAN})$ is unexpectedly $9 \%$ smaller than $A(\mathrm{R}-\mathrm{PAN})$. In other solvents, $A(\gamma$-PAN $)$ is always larger than $A(\mathrm{R}-\mathrm{PAN})$. In DMSO $\gamma$-PAN has an $A$ value $7 \%$ larger than R-PAN, but in $67 \mathrm{wt} \%$ aq nitric acid the reverse is true. Extrapolated $A$ value of $\gamma$ PAN in $56.5 \mathrm{wt} \%$ aq nitric acid (theta solvent) is $4 \%$ larger than the experimentally directly measured value of $A$ for R-PAN in $55 \mathrm{wt} \%$ aq nitric acid (theta solvent). The effect of microtacticity on $A$ of PAN is more remarkable in $67 \mathrm{wt} \%$ aq nitric acid than in DMSO.

For example, $\left\langle S^{2}\right\rangle_{0, z}^{1 / 2}$ for sample cord $\gamma$ $1-3\left(\bar{M}_{w}=16.8 \times 10^{4}\right)$ in theta solvent (i.e., $56.5 \mathrm{wt} \%$ aq nitric acid) was estimated to be $218 \times 10^{-8} \mathrm{~cm}$ (see, Figure 6), and accordingly, $A$ was found to be $1.13 \times 10^{-8} \mathrm{~cm}$. This value is in good agreement with that estimated for $\gamma$-PAN in $56.5 \mathrm{wt}^{\mathrm{o}} \%$ aq nitric acid by method 2C. A values are practically constant in $56 \sim 57$ $\mathrm{wt} \%$ aq nitric acid.

The ratio of $B$ of R-PAN to $\gamma$-PAN in 67 wt $\%$ aq nitric acid is 2.96 and the similar ratio in DMSO is only 1.28 . In other words, $67 \mathrm{wt} \%$ aq nitric acid is a very good solvent for $\mathrm{R}$ PAN, but is not a very good solvent for $\gamma$-PAN at the same temperature. $A$ and $B$ values of $\gamma$ PAN in DMSO are the same as those in 67 wt $\%$ aq nitric acid, respectively, and accord-

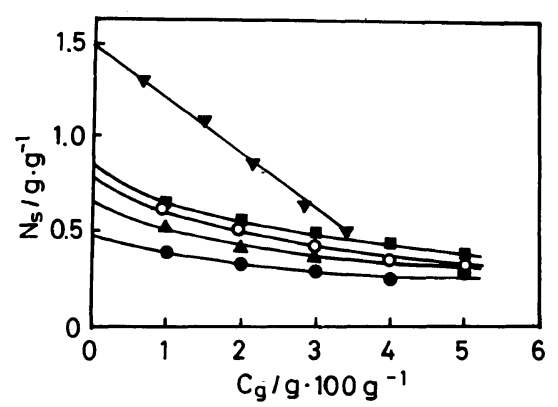

Figure 10 Plots of $N_{\mathrm{s}}$ versus concentration $C_{\mathrm{g}}$ at $25^{\circ} \mathrm{C}$, for $\gamma$-PAN in DMSO (O), R-PAN in DMSO (O), in DMAc ( $(\boldsymbol{\Delta})$, in DMF ( $\mathbf{\square})$, and $67 \mathrm{wt} \%$ aq $\mathrm{HNO}_{3}(\boldsymbol{\Delta})$.

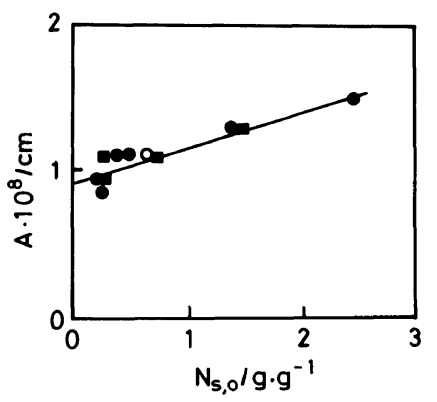

Figure $11 N_{\mathrm{s}, \mathrm{o}}$ dependence of the short-range interaction parameter A for $\gamma$-PAN (O), R-PAN (O) and the acrylonitrile-methyl acrylate copolymer $(\square) .{ }^{32}$

ingly, MHS equations are almost same in the two solvents. For the poor solubility of $\gamma$ PAN, as described before, not only the higher crystallinity, but also low $B$ values are responsible phenomenologically.

Figure 10 shows the dependence of the weight of solvated solvent per gram of polymer $N_{\mathrm{s}}$ on the polymer concentration $C_{\mathrm{g}}(\mathrm{g} /$ $100 \mathrm{~g}$ solution) for $\gamma$-PAN (open mark) and R-PAN (closed mark) in various organic solvents. $N_{\mathrm{s}}$ decreases very gradually with an increase in $C_{\mathrm{g}}$ and there is no significant difference in $N_{\mathrm{s}}$ or $N_{\mathrm{s}, \mathrm{o}}$ of $\left(\equiv \lim _{c_{g} \rightarrow 0} N_{\mathrm{s}}\right)$ between $\gamma$-PAN and R-PAN. Figure 11 shows the dependence of $A$ on $N_{\mathrm{s}, \mathrm{o}}$ of PANs in various solvents. $A$ of $\gamma$-PAN is located just on a common plot for R-PAN and the AN/MA copolymer, obtained in the previous paper. ${ }^{35}$ The solvation is not remarkable even for 
$\gamma$-PAN solution and the larger difference in solubility behavior of these two PAN polymers in organic solvents cannot be interpreted by the solvation concept.

In order to confirm the above conclusions, we investigated the chemical shifts of $\mathrm{CH}$ carbon and $\mathrm{CN}$ carbon of $\gamma-\mathrm{PAN}$ and RPAN in DMSO- $d_{6}$. Peaks of $\mathrm{CH}$ and $\mathrm{CN}$ carbons of $\gamma$-PAN were located at almost the same magnetic field, suggesting that a specific interaction between some functional groups in PAN and the solvent does not depend on the stereospecificity of PAN.

$\gamma$-PAN and R-PAN molecules consist of the same monomer units, but are notably different in steric configurations (Table I). Then, the significant difference in the longrange interaction, expressed by $B$ parameter, between $\gamma$-PAN and R-PAN, cannot be explained if the interaction only occurs between the monomer unit and solvent molecule. We can suppose that the some sequence of monomer units interacts with the solvent molecule and the sequence distribution differences between two polymer types as demonstrated in Table II bring about the large differences in $B$ parameters, especially in 67 $\mathrm{wt} \%$ aq nitric acid. On the 9th and 10th columns of Table VI collects, the steric factor $\sigma$, is defined as

$$
\sigma=A / A_{\mathrm{f}}
$$

(where $A_{\mathrm{f}}$ is $A$ of a hypothetical chain with free internal rotation (model I) and $A_{\mathrm{f}}=0.423 \times$ $10^{-8} \mathrm{~cm}$ for PAN) and the characteristic ratio $C_{\infty}$ is given by

$$
C_{\infty}=A^{2} M_{\mathrm{b}} / l^{2}
$$

(where $M_{\mathrm{b}}$ is the mean molecular weight per skeletal bond $(=26.5)$ and $l$ is the mean bond length $\left(1.54 \times 10^{-8} \mathrm{~cm}\right)$, both calculated from the most probable $A$ value).

Figure 12 shows the effect of the side chain molecular weight on $\sigma$ of the vinyl-type polymers. All the data except for $\gamma$-PAN are cited from the literature. ${ }^{35,43}$ The closed circle is

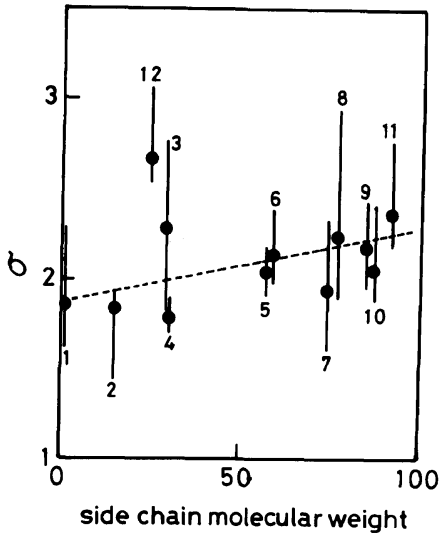

Figure 12. Effect of the molecular weight of the side chain on the conformation parameter $\sigma$ for vinyl-type polymers: ${ }^{43} 1$, polyethylene; 2 , polypropyrene; 3 , poly(1butene); 4, polyisobutene; 5, poly(1-pentene); 6, poly(vinyl acetate); 7, poly(methyl methacrylate); 8, polystyrene; 9, poly(1-octaine), 10, polyisopropyl acrylate; 11 , poly $(\alpha$-methylstyrene); 12 , polyacrylonitrile.

an average value and the full line denotes the range of variation of $\sigma$. Except PAN, $\sigma$ is roughly in linear proportion to the molecular weight of the side chain group, as expressed by a broken line in the figure. R- and $\gamma$-PAN data are located on points much higher than the broken line, indicating that introduction of a $\mathrm{CN}$ group to a vinyl monomer brings about unexpectedly large stiffness in the polymer chain. This stiffness cannot be interpreted by a simple steric hindrance due to the molar volume of the side chain. Dondos and Benoit ${ }^{44}$ showed that dependence of $\sigma$ of the polymer with non-polar side groups, such as. PE, PP, and PS on the molar volume of the side group can be approximately represented by a straight line and $\sigma$ of the polymer with polar side groups like nitrile or alcohol is larger than that of non-polar polymer.

Very recently, Kamide et al. $^{32}$ observed from the ${ }^{13} \mathrm{C}$ NMR spectra of $\gamma$-PAN and R-PAN in DMSO- $d_{6}$ that the intensity ratio of three triad peaks of $\mathrm{CH}$ carbon is just the reverse of that of $\mathrm{CN}$ carbon from a higher magnetic field and then the isotactic triad peak was concluded to shift to a higher mag- 


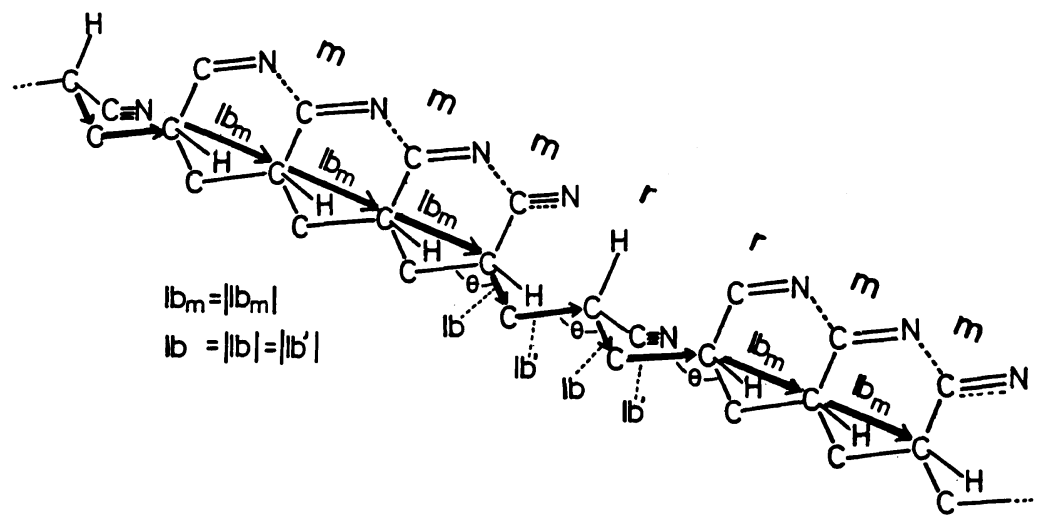

Figure 13. Polyacrylonitrile chain model.

netic field, suggesting isotactic $\mathrm{CH}$ carbon to be strongly shielded as compared with syndiotactic and heterotactic triad $\mathrm{CH}$ carbons. If the $\mathrm{CN}$ group is free and triple bond exists between $\mathrm{C}$ and $\mathrm{N}$, such a strong shield effect cannot be expected. In other words, the shielding effect is caused only in the case when $\mathrm{CN}$ groups have a double bond nature, and the meso part of PAN may have a transtrans or almost trans-trans conformation. The former conformation might stiffen the meso sequence in the solution. Additional experimental facts supporting this idea are (1) the lower magnetic field component of $\mathrm{CN}$ carbon peaks becomes sharp and (2) there is a specific interaction of $\mathrm{Cu}^{2+}$ ion with $\mathrm{CN}$ group in the meso sequence. ${ }^{45}$

If the above-mentioned conclusion is acceptable, it is expected that $\gamma$-PAN is inflexible as compared with R-PAN. We can consider a hypothetical PAN chain, in which the meso sequence can be regarded as, at least partly, a rigid rod and the racemo sequence is freely rotatable. This chain is an alternative, extreme model (model II) for a hypothetical chain. Here, we assume that "blocks" consisting of less than $n$ meso sequences $\left(m_{1} m_{1} m_{2}\right.$, $\left.m_{1} m_{2} m_{3}, \cdots, m_{1} \cdots m_{n}\right)$ are totally rigid rod and "blocks" consisting of a larger number of meso sequences than $n$ can be divided into two or more rods with free rotation, whose number of meso sequences does not exceed

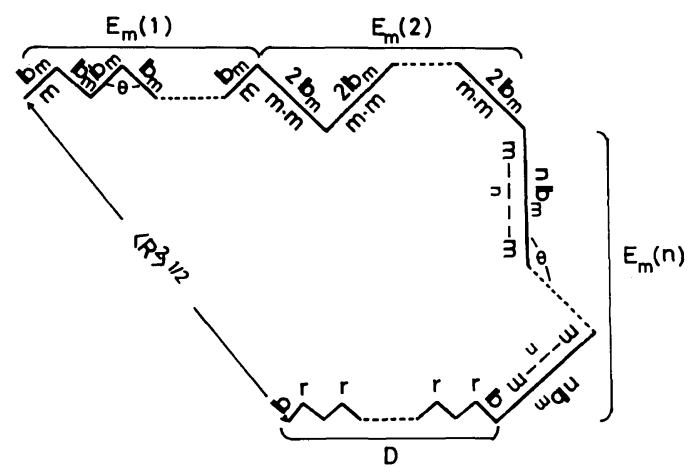

Figure 14. $\left\langle R^{2}\right\rangle^{1 / 2}$ of model chain II.

$n$ (see, Figures 13 and 14).

Here, we used the data in Table II for calculation of $A_{\mathrm{f}}$ of model II. For model II chain $A_{\mathrm{f}, \mathrm{II}}$ is given by

$$
A_{\mathrm{f}, \mathrm{II}}=\left\langle R^{2}\right\rangle_{\mathrm{f}, \mathrm{II}}^{1 / 2} / M^{1 / 2}
$$

Consider a polymer chain consisting of $L$ segments and represent vector of a segment constituting a polymer chain with $\boldsymbol{u}_{i}(i=1$, $\cdots, L)$. The end-to-end distance vector $\boldsymbol{R}$ of the chain is

$$
\boldsymbol{R}=\sum_{i=1}^{L} \boldsymbol{u}_{i}
$$

and the mean-square end-to-end distance $\left\langle R^{2}\right\rangle$ is given by 


$$
\left\langle R^{2}\right\rangle=\sum_{i=1}^{L} l_{i}^{2}+2 \sum_{\substack{i=1 \\(i<j)}}^{L} \sum_{\substack{j=1 \\(i<j}}^{L} l_{i} l_{j}(-\cos \theta)^{j-i}
$$

if the chain permits a free internal rotation. Here, $l_{i}=\left|\boldsymbol{u}_{i}\right|, l_{j}=\left|\boldsymbol{u}_{j}\right|$ and $\theta$ is the bond angle.

In Figure $14,\left\langle R^{2}\right\rangle_{\text {of ,II }}$ in equation 17 is given by (see Appendix)

$$
\begin{aligned}
\left\langle R^{2}\right\rangle_{\mathrm{f}, \mathrm{II}}= & \sum_{x=1}^{n} E_{m}(x)\left(x b_{m}\right)^{2}+D b^{2} \\
& +2 \sum_{x=1}^{n}\left[\sum_{i=1}^{E_{m}(x)} \sum_{j=1}^{E_{m}(x)}\left(x b_{m}\right)^{2}(-\cos \theta)^{j-i}\right] \\
& +2 \sum_{i=1}^{D} \sum_{j=1}^{D} b^{2}(-\cos \theta)^{j-i} \\
& +2 \sum_{x=1}^{n} \sum_{y=1}^{n}\left[\sum_{i=1}^{\left.E^{2}<j\right)} \sum_{j=1}^{E_{m}(x)}\left(x b_{m}\right)\left(y b_{m}\right)\right. \\
& \left.\times(-\cos \theta)\left(j-i+\sum_{n=x}^{(i<j)} E_{m}(h)\right)\right] \\
& +\sum_{x=1}^{n}\left[\sum_{i=1}^{E_{m}(x)} \sum_{j=1}^{D} b\left(x b_{m}\right)\right. \\
& \left.\times(-\cos \theta)\left(j-i+\sum_{n=x}^{n} E_{m}(h)\right)\right]
\end{aligned}
$$

Where

$$
D=\sum_{q=1}^{q^{\prime}}\left(2 q \overline{\left.F_{r}(q)\right)}\right.
$$

(total number of $b$ bond vector)

and

$$
E_{m}(h)=\sum_{k=0}^{k^{\prime}} \overline{F_{m}(k n+h)} \quad\left(\text { for } h=1, \cdots, g^{\prime}\right)
$$

$$
\begin{aligned}
=\sum_{k=0}^{k^{\prime}} \frac{}{F_{m}(k n+h)}-\overline{F_{m}\left(p^{\prime}\right)} \\
\quad\left(\text { for } h=g^{\prime}+1, \cdots, n-1\right)
\end{aligned}
$$

$$
\begin{aligned}
E_{m}(n)= & \sum_{k=0}^{k^{\prime}} \sum_{h=0}^{n-1} k \overline{F_{m}(k n+h)} \\
& -\sum_{h=g^{\prime}+1}^{n-1} k^{\prime} \overline{F_{m}\left(k^{\prime} n+h\right)}
\end{aligned}
$$

$b_{m}$ is the magnitude of vector $\boldsymbol{b}_{m}$ connecting two neighboring $\mathrm{CN}$ carbons in a meso sequence (Figure 13) $\left(\boldsymbol{b}_{m}=\left|\boldsymbol{b}_{m}\right|\right), b$ is the magnitude of bond vector $\boldsymbol{b}$ in a main chain $(b=|\boldsymbol{b}|), \theta$ is the bond angle, $q^{\prime}$ is the largest number of $q$ existing in a chain. In deriving eq 17 , we assumed (i) meso sequence $m_{1} m_{2}$ $\cdots m_{p}$ can be divided into $k$ rigid rod blocks, each consisting of meso sequence $m_{1} m_{2} \cdots m_{n}$, and a rigid rod block $m_{1} m_{2} \cdots m_{g}$;

$p=k n+g\left(k=1, \cdots, k^{\prime} ; g=0, \cdots, n-1\right)$

we define $g^{\prime}\left(\equiv p^{\prime}-k^{\prime} n\right)$ as $g$ for maximum $p\left(p^{\prime}\right)$ in a chain, (ii) a block $m_{1} m_{2} \cdots m_{x}(x \leqq$ $n$ ) can be expressed by single bond vector $x \boldsymbol{b}_{m}$, (iii) a racemo configuration can be represented by two bond vectors $\boldsymbol{b}$ and $\boldsymbol{b}^{\prime}$ (Figure 13) with the same magnitude $b(|\boldsymbol{b}|=$ $\left|\boldsymbol{b}^{\prime}\right|=b$ ), (iv) eàch bond angle between two arbitrarily chosen bond vector is the same, (v) the chain is an absolutely free rotating chain. $D$ and $E_{m}(h)$, given in eq 18 and 19 , give the total number of $\boldsymbol{b}$ vectors and $h \boldsymbol{b}_{m}$ vectors.

In eq 17 , the first, second, third and fourth terms of the right-hand side are the scalar products of the meso bond vector $\left(x \boldsymbol{b}_{m}\right)$, racemo bond vector $\boldsymbol{b}$, bond vector of a rigid meso block $m_{1} m_{2} \cdots m_{x}\left(x \boldsymbol{b}_{m}\right)$, and bond vector $\boldsymbol{b}$, respectively. The fifth term is the scalar product between two rigid meso blocks with different $x$ and the last term is the scalar product between $\boldsymbol{b}_{m}$ and $\boldsymbol{b}$. Here, $b_{m}=2.516 \AA, b=1.54 \AA$, and $\theta=109.5$ were employed.

First, $D, E_{m}(h)$ and $E_{m}(n)$ were calculated by putting the values of $\overline{F_{m}(p)}$ and $\overline{F_{r}(q)}$ in Table II into eq $18,19 \mathrm{a}, \mathrm{b}$, and $\mathrm{c}$. Using the values of $D, E_{m}(h)$ and $E_{m}(n)$, thus calculated, $\left\langle R^{2}\right\rangle_{\mathrm{f}, \mathrm{II}}$ was calculated from eq $17, A_{\mathrm{f}, \mathrm{II}}$ and $\sigma$ were readily estimated from 14 and 12 , respectively. The following quantities were, for example, 


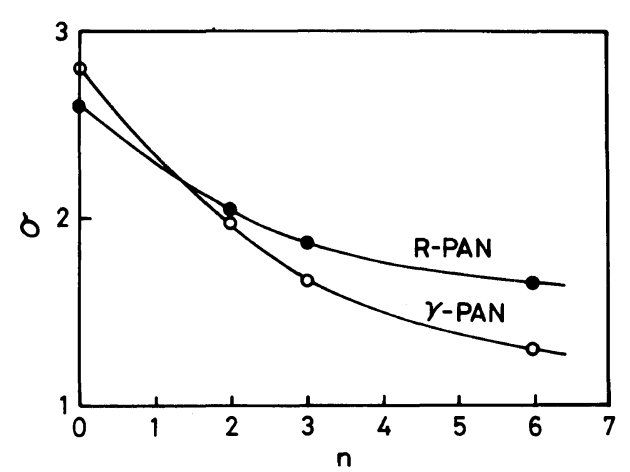

Figure 15. Plot of the conformation parameter $\sigma$ as a function of the length of rigid rod meso-blocks $n$.

obtained: For $\gamma$-PAN, $D=2818, E_{m}(1)=318$, $E_{m}(2)=1273$; for R-PAN, $D=3860, E_{m}(1)=$ $657, E_{m}(2)=716$ at $n=2$.

Figure 15 shows $\sigma\left(\equiv A / A_{\mathrm{f}, \mathrm{II}}\right)$ as a function of $n$ for $\gamma$ - and R-PAN. Evidently, $\sigma$ for both polymers decreases monotonously with an increase in $n$ (i.e., the rigidity of a meso sequence). When no additional effect, besides the steric hindrance due to the molar volume of the side group, exists, $\sigma$ for PAN is roughly estimated from Figure 12 to be 2 . Reversely, we can evaluate $n$ to be $\sim 2$ for $\gamma$ - and RPAN from $\sigma \cong 2$.

Summarizing (1) $\gamma$-PAN does not have the same MHS equations as R-PAN, even in 67 $\mathrm{wt} \%$ aq nitric acid and DMSO. In these solvents, the exponent $a$ is not larger than 0.6. In this sense, there is no true good solvent for $\gamma$-PAN. (2) For R-PAN, it is expected that DMF is a better solvent than DMSO. But $\gamma$-PAN does not dissolve, at least, at room temperature in DMF. (3) A $56.5 \mathrm{wt} \%$ aq nitric acid is a theta solvent for $\gamma$-PAN and in that mixture both $[\eta]$ and $\left\langle S^{2}\right\rangle_{z}^{1 / 2}$ are proportional to $\bar{M}_{w}^{1 / 2}$. (4) In DMSO, $A_{2}$ for R-PAN is larger than that for $\gamma$-PAN when compared at the same $\bar{M}_{w}$. (5) The unperturbed chain dimension $A$ is larger for $\gamma$-PAN than for R-PAN.

\section{APPENDIX}

Derivation of $E q 17 \sim 19$

We assume that the polymer chain is an assemble of $E_{m}(h)$ rigid meso blocks $m_{1} m_{2}$ $\cdots m_{h}(h=1,2, \cdots, n)$ and $E_{r}(q)$ racemo blocks $r_{1} r_{2} \cdots r_{q}\left(q=1,2, \cdots, q^{\prime}\right)$, both arranged linearly. Equation 16 does not change independently of the permutation of the assemble. We define $L(i)=\left|l_{i}\right|$ and rearrange $l_{i}$ from $i=1$ to $\sum_{h=1}^{n} E_{m}(h)+D$ in the following manner.

$$
\begin{aligned}
& L(1)=L(2)=\cdots=L\left(E_{m}(1)\right)=b_{m} \\
& L\left(E_{m}(1)+1\right)=\cdots=L\left(\sum_{h=1}^{2} E_{m}(h)\right)=2 b_{m} \\
& \quad \cdots \\
& L\left(\sum_{h=1}^{n-1} E_{m}(h)+1\right)=\cdots=L\left(\sum_{h=1}^{n} E_{m}(h)\right)=n b_{m} \\
& L\left(\sum_{h=1}^{n} E_{m}(h)+1\right)=\cdots=L\left(\sum_{h=1}^{n} E_{m}(h)+D\right)=b
\end{aligned}
$$

Then, the first term in the right-hand side of eq 16 can be devided into two parts (see Figure 13):

(i) for $l_{i}=x b_{m}$

$$
\sum_{x=1}^{n} E_{m}(x)\left(x b_{m}\right)^{2}
$$

(ii) for $l_{i}=b$

$$
\sum_{x=1}^{q^{\prime}} 2\left(x \overline{F_{r}(x)}\right) b^{2}=D b^{2}
$$

and the second term in the right-hand side of eq 16 can be devided into four parts:

(iii) for $l_{i}=l_{j}=x b_{m}(x=1, \cdots, n)$ 


\section{K. KAMIDE, H. YAMAZAKI, and Y. MiYAZAKI}

$$
\begin{aligned}
& 2 \sum_{i=1}^{E_{m}(1)} \sum_{j=i}^{E_{m}(1)}\left(1 b_{m}\right)\left(1 b_{m}\right)(-\cos \theta)^{j-i} \\
& +2 \sum_{i=1}^{E_{m}(2)} \sum_{j=1}^{E_{m}(2)}\left(2 b_{m}\right)\left(2 b_{m}\right)(-\cos \theta)^{\left\{\left(j+E_{m}(1)-\left(i+E_{m}(1)\right)\right\}\right.} \\
& +\cdots \\
& +2 \sum_{i=1}^{E_{m}(n)} \sum_{j=1}^{E_{m}(n)}\left(n b_{m}\right)\left(n b_{m}\right)(-\cos \theta)\left\{\left(j+\sum_{n=1}^{n-1} E_{m}(h)\right)-\left(i+\sum_{n=1}^{n-1} E_{m}(h)\right)\right\} \\
& \quad=2 \sum_{x=1}^{n}\left\{\sum_{i=1}^{E_{m}(x)} \sum_{j=1}^{E_{m}(x)}\left(x b_{m}\right)(-\cos \theta)^{(i-j)}\right\}
\end{aligned}
$$

(iv) for $l_{i}=l_{j}=b$

$$
\begin{aligned}
& 2 \sum_{i=1}^{D} \sum_{j=1}^{D} b \cdot b(-\cos \theta)\left\{\left(j+\sum_{h=1}^{n} E_{m}(h)\right)-\left(i+\sum_{h=1}^{n} E_{m}(h)\right)\right\} \\
& \quad=2 \sum_{i=1}^{D} \sum_{j=1}^{D}(-\cos \theta)^{(j-i)}
\end{aligned}
$$

(v) for $l_{i}=x b_{m}$ and $l_{j}=y b_{m},(x<y ; x, y=1, \cdots, n)$

$$
\begin{aligned}
& 2 \sum_{i=1}^{E_{m}(1)} \sum_{j=1}^{E_{m}(2)}\left(1 b_{m}\right)\left(2 b_{m}\right)(-\cos \theta)^{\left(j+E_{m}(1)-i\right)} \\
& +\cdots \\
& +2 \sum_{i=1}^{E_{m}(1)} \sum_{j=1}^{E_{m}(n)}\left(1 b_{m}\right)\left(n b_{m}\right)(-\cos \theta)\left(j+\sum_{h=1}^{n-1} E_{m}(h)-i\right) \\
& +2 \sum_{i=1}^{E_{m}(2)} \sum_{j=1}^{E_{m}(3)}\left(2 b_{m}\right)\left(3 b_{m}\right)(-\cos \theta)\left\{\left(j+\sum_{h=1}^{3-1} E_{m}(h)\right)-\left(i+\sum_{h=1}^{2-1} E_{m}(h)\right)\right\} \\
& +\cdots \\
& +2 \sum_{i=1}^{E_{m}(2)} \sum_{j=1}^{E_{m}(n)}\left(2 b_{m}\right)\left(n b_{m}\right)(-\cos \theta)\left\{\left(j+\sum_{h=1}^{n-1} E_{m}(h)\right)-\left(i+\sum_{h=1}^{2-1} E_{m}(h)\right)\right\} \\
& +\cdots \\
& +2 \sum_{i=1}^{E_{m}(n-1)} \sum_{j=1}^{E_{m}(n)}\left\{(n-1) b_{m}\right\}\left(n b_{m}\right)(-\cos \theta)\left\{\left(j+\sum_{h=1}^{n-1} E_{m}(h)\right)-\left(i+\sum_{h=1}^{(n-1)-1} E_{m}(h)\right)\right\} \\
& =2 \sum_{x=1}^{n} \sum_{y=1}^{n}\left\{\sum_{i=1}^{E_{m}(x)} \sum_{j=1}^{E_{m}(y)}\left(x b_{m}\right)\left(y b_{m}\right)(-\cos \theta)\left(j-i+\sum_{h=x}^{Y-1} E_{m}(h)\right)\right\}
\end{aligned}
$$


(vi) for $l_{i}=x b_{m}$ and $l_{j}=b(x=1, \cdots, n)$

$$
\begin{aligned}
& \sum_{i=1}^{E_{m}(1)} \sum_{j=1}^{D} b\left(1 b_{m}\right)(-\cos \theta)\left(j+\sum_{h=1}^{n} E_{m}(h)-i\right) \\
& +\sum_{i=1}^{E_{m}(2)} \sum_{j=1}^{D} b\left(2 b_{m}\right)(-\cos \theta)\left\{\left(j+\sum_{h=1}^{n} E_{m}(h)\right)-\left(i+\sum_{h=1}^{2-1} E_{m}(h)\right)\right\} \\
& +\ldots \\
& +\sum_{i=1}^{E_{m}(n)} \sum_{j=1}^{D} b\left(n b_{m}\right)(-\cos \theta)\left\{\left(j+\sum_{h=1}^{n} E_{m}(h)\right)-\left(i+\sum_{h=1}^{n-1} E_{m}(h)\right)\right\} \\
& =\sum_{x=1}^{p}\left\{\sum_{i=1}^{E_{m}(x)} \sum_{j=1}^{D} b\left(x b_{m}\right)(-\cos \theta)\left(j-i+\sum_{h=x}^{n} E_{m}(h)\right)\right.
\end{aligned}
$$

Summation of eq A·1 eq A.6 gives eq 17 . By putting $n=p^{\prime}$ in eq $19 \mathrm{a}$ and $19 \mathrm{~b}$, we obtain $E_{m}(h)=\overline{F_{m}(h)}$ and, by putting $k^{\prime} \rightarrow \infty$ $\left(p^{\prime} \rightarrow \infty\right)$, we obtain $\overline{F_{m}\left(p^{\prime}\right)} \rightarrow 0$. In the case of $k^{\prime} \rightarrow \infty$, eq $19 \mathrm{a}$ and $19 \mathrm{~b}$ reduces to

$$
E_{m}(h)=\sum_{k=0}^{\infty} \overline{F_{m}(k p+h)} \quad(h=1, \cdots, p)
$$

\section{REFERENCES}

1. J. B. Kinsinger and R. E. Hughes, J. Phys. Chem., 63, 2002 (1959).

2. P. Parrini, F. Sebastiano, and G. Messina, Makromol. Chem., 38, 27 (1960).

3. J. B. Kinsinger and R. E. Hughes, J. Phys. Chem., 67, 1922 (1963)

4. H. Inagaki, T. Miyamoto, and S. Ohta, J. Phys. Chem., 70, 3420 (1966).

5. A. Nakajima and A. Saigo, J. Polym. Sci., 6, 735 (1968).

6. I. Sakurada, A. Nakajima, O. Yoshizaki, and K. Nakamae, Kolloid Z.-Z. Polym. B, 186, 41 (1962).

7. S. Krause and E. Cohn-Ginsberg, Polymer, 3, 565 (1962).

8. S. Krause and E. Cohn-Ginsberg, J. Phys. Chem., 67, 1479 (1963).

9. G. V. Schulz, W. Wunderlich, and R. Kirste, Makromol. Chem., 75, 22 (1964).

10. F. Danusso and G. Moraglio, J. Polym. Sci., 24, 161 (1957).

11. W. R. Krigbaum, D. K. Capenter, and S. Newman, J. Phys. Chem., 62, 1586 (1958).
12. L. Trossarelli, E. Campi, and G. Saini, J. Polym. Sci., 35, 205 (1959).

13. J. M. G. Cowie, B. Bywater; and D. J. Worsfold, Polymer, 8, 105 (1967).

14. J. M. G. Cowie and S. Bywater, J. Polym. Sci., 6, 499 (1968).

15. T. Kato, K. Miyaso, I. Noda, T. Fujimoto, and M. Nagasawa, Macromolecules, 3, 777 (1970).

16. T. Kato, K. Miyaso, I. Noda, T. Fujimoto, and M. Nagasawa, Macromolecules, 3, 787 (1970).

17. W. R. Krigbaum, J. E. Kurz, and P. Smith, J. Phys. Chem., 65, 1984 (1961).

18. K. Satynarayana, S. Astry, and R. D. Patel, Eur. Polym. J., 5, 79 (1969).

19. G. Moraglio and J. Brzezinski, J. Polym. Sci., B, 2, 1105 (1964).

20. G. Moraglio and G. Gianotti, Eur. Polym. J., 5, 781 (1969).

21. J. E. Mark, R. A. Wessling, and R. E. Hughes, $J$. Phys. Chem., 70, 1895 (1966).

22. J. E. Mark, R. A. Wessling, and R. E. Hughes, J. Phys. Chem., 70, 1903 (1966).

23. M. Matsumoto and Y. Ohyanagi, J. Polym. Sci., 37, 558 (1959).

24. K. Kamide, Kobunshi, 11, 49 (1962).

25. W. R. Krigbaum, "Newer Method of Polymer Characterization," B. Ke, Ed., John Willey \& Sons, New York, N. Y., 1964, Chapter 1.

26. J. M. G. Cowie "Light Scattering from Polymer Solution," M. B. Huglin, Ed., Academic Press, New York, N. Y., 1972, Chapter 14.

27. H. Inagaki, K. Hayashi, and T. Matsuo, Makromol. Chem., 84, 80 (1965).

28. K. Kamide and T. Terakawa, Makromol. Chem., 155, 25 (1972).

29. K. Matsuzaki, T. Uryu, M. Okada, and H. Shiroki, J.P Polym. Sci., A-1, 6, 1475 (1968). 
30. K. Matsuzaki, T. Uryu, M. Okada, and H. Shiroki, J. Polyn. Sci., A-1, 9, 1701 (1971).

31. K. Kamide, H. Kobayashi, Y. Miyazaki, and C Nakayama, Kobunshi Kagaku, 24, 679 (1967).

32. K. Kamide, H. Yamazaki, K. Okajima, and K. Hikichi, Polym. J., 17, 1233 (1985).

33. K. Kamide, H. Yamazaki, K. Okajima, and K. Hikichi, Polym. J., 17, 1291 (1985).

34. D. M. White, J. Am. Chem. Soc., 82, 5678 (1960).

35. K. Kamide, Y. Miyazaki, and H. Kobayashi, Polym. J., 17, 607 (1985).

36. Y. Fujisaki and H. Kobayashi, Kobunshi Kagaku, 19, 49 (1962)

37. H. Yamazaki, K. Kamide, and Y. Miyazaki, to be submitted to Polym. J.

38. M. Kurata, M. Fukatsu, H. Sotobayashi, and H.
Yamakawa, J. Chem. Phys., 41, 139 (1964).

39. M. Fixman, J. Chem. Phys., 36, 3123 (1962).

40. H. Baumann, J. Polym. Sci., Polym. Lett. Ed., 3, 1069 (1965).

41. W. H. Stockmayer and M. Fixman, J. Polym. Sci., C, 1, 137 (1963).

42. K. Kamide and W. Moore, J. Polym. Sci., Polym. Lett. Ed., 2, 1029 (1964).

43. M. Kurata, Y. Tsumashima, M. Iwata, and K. Kamata, "Polymer Handbook," 2nd ed, J. Brandrup and E. H. Immergut, Ed., John Wiley \& Sons, New York, N. Y., 1975.

44. A. Dondos and H. Benoit, Macromolecules, 4, 279 (1971).

45. K. Kamide, H. Yamazaki, K. Okajima, and K. Hikichi, Polym.J., 18, 277 (1986). 